Short reports

\title{
Management of the extrapyramidal manifestations of phenylketonuria with L-dopa
}

\author{
M D MACLEOD, J F MUNRO, J G LEDINGHAM, AND J W FARQUHAR \\ Medical Unit, Eastern General Hospital, and The Royal Hospital for Sick Children, Edinburgh
}

SUMMARY We report on a patient with phenylketonuria who developed disabling extrapyramidal features that were successfully controlled with L-dopa. We recommend that all such patients are given a trial with this drug.

Inadequately treated phenylketonuria (PKU) is commonly associated with severe mental retardation ${ }^{1}$ and often with various neurological complications. Some of these are suggestive of parkinsonism. ${ }^{2}$ This report describes the long term effect of L-dopa in such a patient.

\section{Case report}

A girl was born in May 1959. In October 1960, before the national routine neonatal screening for phenylketonuria began, she was referred to hospital with pallor and a haemoglobin value of $6.8 \mathrm{~g} / \mathrm{dl}$. The anaemia responded to treatment with oral iron, but she remained unwell and unable to stand without support. In December 1960, after further investigations she was found to be suffering from PKU. The serum phenylalanine concentration was 1996 $\mu \mathrm{mol} / 1 \mathrm{l}(33 \mathrm{mg} / 100 \mathrm{ml})$. Dietary restriction was introduced, and when estimated again in July 1961 her phenylalanine concentration was consistently less than $181 \mu \mathrm{mol} / 1(3 \mathrm{mg} / 100 \mathrm{ml})$. She was able to stand but was still unable to walk. Hospital attendance was erratic but she improved slowly.

Her mother insisted that dietary adherence was strict until she was aged 6 years, when her IQ was assessed at 66. Thereafter, on an unrestricted diet she made further progress while attending a special schuol for the mentally handicapped.

She was lost from hospital follow up until April 1973 when she was referred because of increasing disability arising from a tremor, mainly affecting the right arm, of such severity that she had great difficulty in writing, in drinking without spilling, and was unable to do needlework. On examination she had a marked intention and postintention tremor, affecting the right arm more than the left. In June 1973 treatment with L-dopa began, increasing to a maximum tolerated dose of $187 \mathrm{mg} /$ day. Treatment was associated with considerable subjective improvement. In May 1974 the L-dopa was replaced with Sinemet-275, 8 tablets daily. Formal assessment performed 3 years after treatment started showed clear improvement in her ability to write and she could drink without spilling and undertake fine activities such as needlework. Since then her condition has changed little. She has become more assured in her personal relationships, plans to marry in the near future, and has a job in a laundry. On one occasion treatment was discontinued inadvertently for several weeks and its withdrawal was associated with a recurrence of her symptoms.

\section{Discussion}

In the untreated patient with PKU the severity of neurological damage depends on the degree of the biochemical disturbance. Permanent central nervous system impairment can be minimised by early diagnosis and by strict dietary control. Relaxation of dietary restriction after several years is widely recommended ${ }^{3}$ but the efficacy of this policy has yet to be fully established.

Tyrosine-3-hydroxylase is responsible for the conversion of tyrosine to L-dopa and other biogenic amines. Its concentration seems to depend on the concentration of phenylalanine in the plasma and the tissues. ${ }^{4}$ Curtius et al. ${ }^{4}$ suggested that the inhibition of L-dopa formation might play an important part in the pathogenesis and neurological complications of phenylketonuria.

Our patient's previous disabling neurological features have now been successfully controlled for the past 8 years by treatment with L-dopa and thereafter Sinemet 275. Our observations confirm those of the only other reported case. We recommend that all patients suffering from the neurological sequelae 
of phenylketonuria should be given a trial with L-dopa.

\section{References}

1 Farquhar JW, Richmond J, Tait HP. Phenylketonuria in pediatric practice. Clin Pediatr (Phila) 1963; 2: 504-16.

2 Payne RS. The variability in manifestations of untreated patients with phenylketonuria (phenylpyruvic aciduria). Pediatrics 1957; 20: 290-302.

3 Anonymous. The duration of treatment of phenylketonuria. Lancet 1974; i : 971-2.
4 Curtius H-cH, Baerlocher K, Völlmin JA. Pathogenesis of phenylketonuria: inhibition of dopa and catecholamine synthesis in patients with phenylketonuria. Clin Chim Acta 1972; 42: 235-9.

Correspondence to Dr Michael MacLeod, Medical Unit, Eastern General Hospital, Seafield Street, Edinburgh EH6 7LN.

Received 11 January 1983

\title{
Effects of artificial surfactant on lung function and blood gases in idiopathic respiratory distress syndrome
}

\author{
A D MILNER, H VYAS, AND I E HOPKIN \\ Department of Child Health, City Hospital, Nottingham
}

SUMMARY We have investigated the effect of giving two $25 \mathrm{mg}$ doses of dry surfactant powder to the airways of 10 preterm babies with severe idiopathic respiratory distress syndrome requiring intermittent positive pressure ventilation. No useful change in static total compliance or blood gases was seen. We conclude that dry surfactant powder does not have a role in the management of severe idiopathic respiratory distress syndrome.

Recent studies from $\mathrm{Japan}^{1}$ and Canada ${ }^{2}$ suggested that giving artificial surfactant may improve rapidly and dramatically the clinical condition of babies with severe idiopathic respiratory distress syndrome (IRDS) requiring ventilatory support. These were, however, anecdotal investigations, and the only well controlled trial reported so far failed to show that the treatment altered blood gas values or clinical state to any appreciable extent. ${ }^{3}$ For this reason we have completed a trial examining the effects of artificial surfactant, delivered to the respiratory tract as a dry powder on static compliance and transcutaneous oxygen and carbon dioxide in preterm babies with severe IRDS.

\section{Method}

Ten preterm babies with a mean gestational age of 30.1 weeks (range 27-33 weeks) and a mean birthweight of $1.45 \mathrm{~kg}$ (range $0.77-1.86 \mathrm{~kg}$ ) were investigated. All had a clinical diagnosis of IRDS with typical chest $x$-ray findings and a negative shake test on admission to the neonatal unit. Their mean age at the time of study was $17 \cdot 3$ hours (range 6-29 hours) and they had all required ventilation for at least 5 hours. Six were born by normal spontaneous vertex delivery and 4 by caesarean section. Their ventilator settings were mean inflation pressures $23 \cdot 1 / 0 \mathrm{~cm} \mathrm{H}_{2} \mathrm{O}$, (range $15-35 / 0 \mathrm{~cm} \mathrm{H}_{2} \mathrm{O}$ ) at a rate of 42.4 (range 24-60 per minute); an inspiratory: expiratory ratio of $1: 1$ (range $0 \cdot 7-1: 1-1.5$ ); and an inspired oxygen of $71.5 \%$ (range $50-100 \%$ ). These settings were left unchanged throughout the study.

Inflation pressure was measured using a strain gauge pressure transducer connected to the endotracheal tube by a 21 gauge butterfly needle. This was calibrated against a water column at the end of each investigation. Changes in thoracic volume were recorded using an inflated rubber jacket which encompassed the chest and abdomen and was then inflated to a pressure of $2 \mathrm{~cm} \mathrm{H}_{2} \mathrm{O}$. Tidal exchange produced pressure swings in the jacket of less than $1 \mathrm{~mm} \mathrm{H}_{2} \mathrm{O}$. Calibration was achieved by injecting and withdrawing $20 \mathrm{ml}$ aliquots of air into and out of the jacket at intervals of 4 to 5 breaths and measuring the change in end-tidal baseline. ${ }^{4}$ All signals were collected on an FM tape recorder (Racal) and subsequently relayed onto ultraviolet sensitive paper for analysis. Transcutaneous carbon dioxide and usually transcutaneous oxygen were monitored throughout using the Radiometer transcutaneous blood gas system with the 2 probes attached to the abdominal skin under the jacket. In 3 babies arterial oxygen was monitored throughout using the Searle intra-arterial oxygen electrode and the transcutaneous oxygen measurements were then omitted.

Once the jacket, probes, and transducers were in position measurements of transcutaneous oxygen 